\title{
A Robust Hybrid Algorithm for Image Watermarking
}

\author{
Sharanjit Singh ${ }^{1}$ and Neha Mahant ${ }^{2}$ \\ ${ }^{1}$ Assistant Professor, ${ }^{2} \mathrm{PG}$ Student \\ Department of Computer Science, Regional Campus, Gurdaspur, Punjab, India \\ E-Mail: nehamahant74@gmail.com
}

\begin{abstract}
Image watermarking is the mechanism of hiding the critical information on the segment of the image that can either be least significant or most significant in nature. Image watermarking involves multiple images that are merged together to achieve a common image that is transferred over a digital medium. Proposed system uses slant let transformation to achieve better result of image security in terms of accuracy. Accuracy is achieved by minimising mean square error and improving peak signal to noise ratio.

Keywords: Image watermarking, Slant let Transformation. PSNR, MSE
\end{abstract}

\section{INTRODUCTION}

In present days, the assurance and illicit redistribution of advanced media has turned into a noteworthy issue. The advanced watermarking has been utilized to shield computerized data from unlawful redistribution and changes[1]. In advanced water denoting the image has been improved by installing clamour tolerant flag into bearer flag. Late years have seen a quick development in the accessibility of computerized media content. Today, computerized media archives can be conveyed by means of the World Wide Web to countless without much exertion and cash. Moreover, not at all like conventional simple replicating, with which the nature of the copied content is corrupted, advanced apparatuses can without much of a stretch create extensive measure of ideal duplicates of computerized archives in a brief period. This simplicity of computerized interactive media appropriation over the Internet, together with the likelihood of boundless duplication of this information, debilitates the protected innovation rights like never before. Hence, content proprietors are energetically looking for advances that guarantee to ensure their rights.

In the present period [2], [3] advanced security turns into the most smoking point because of its capacity to decrease the cost related with registering. Computerized registering gives the on request benefits like stockpiling, servers, assets and so on to the clients without physically obtaining them and the installment is as per pay per utilize. Since image processing gives the capacity, diminishes the overseeing expense and time for association to the client however security and classification turns into the one of the greatest problems before us. To tackle the issue [4] slantlet transformation is used. The real issue with cloud condition is the quantity of client is transferring their information on distributed storage so now and again because of absence of security there might be odds of loss of privacy. To beat these hindrances an outsider is required to anticipate information, information encryption, and trustworthiness and control unapproved access for information stockpiling to the cloud.

With the fast improvement of equipment and programming computerized security acquires the insurgency the business. It gives assets like computational power, stockpiling, calculation stage promotion applications to client on request through web. A portion of the cloud suppliers are Amazon, IBM, Google, Sales drive, Microsoft and so on. Computerized processing highlights included asset sharing, multi-tenure, remote information stockpiling and so on yet it challenges the security framework to secure, ensure and process the information which is the property of the individual, undertakings and governments[5]. Despite the fact that, there is no prerequisite of information or ability to control the foundation of mists; it is dynamic to the client. It is an administration of an Internet with high adaptability, nature of administration, higher throughput and high processing power. Advanced registering suppliers send normal online business applications which are gotten to from servers through web program. Information security is the greatest issue in computerized security and it is difficult to determine it.

Watermarking through the advancement of DWT known as slant let transformation is proposed[6], [7]. The logo image and main image are collaborated together by the use of slantlet transformation. Singular valued decomposition is used to reduce the complexity of operation. The entire image is decomposed into three parts. Matrix indicated with $\mathrm{S}, \mathrm{V}$ and $\mathrm{D}$. all the colour dissimilarities are denoted with $\mathrm{D}$ and intensity mismatch is resolved with singular matrix $\mathrm{s}$ and $v$. Flow of proposed system is given as under.

Basic principal of watermarking is given as under

1. Input the image (primary image)

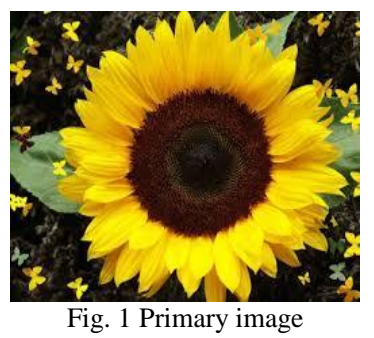


2. Input the logo image(Secondary image)

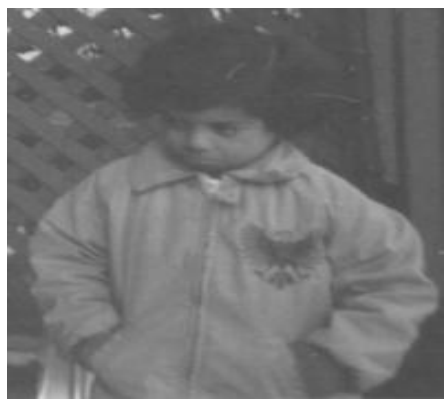

Fig. 2 Secondary image

3. Apply the mechanism of watermarking to merge the two images.

4. Output the watermarked image

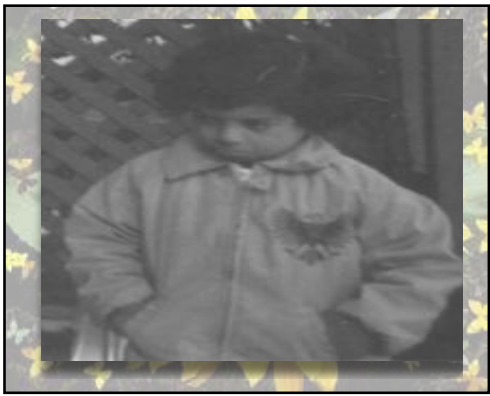

Fig. 4 watermarked image

Obtain the parameters such as PSNR and MSE for performance measurement of techniques used. Next section gives the brief overview of existing security techniques used within the digital systems.

\section{TECHNIQUES USED FOR IMAGE SECURITY}

To achieve the image security, watermarking and stenography mechanisms commonly followed. The techniques for image security are described as under

\section{A. LSB Stenography}

In LSB stenography, the least significant bits of the image are chosen and replaced with the logo image[8]. The contrast enhancement mechanism is implied in order to change the contrast of both the images so that merged images are clearly visible. Problem with this approach is however those attackers easily can determine the position of the logo and hence attack can easily takes place. In order to tackle the issue, MSB stenography is followed.

\section{B. MSB Stenography}

In MSB stenography, most significant bits are enriched with the logo image and hence merged image is obtained[9]. The assumption is that MSB are less prone to attacks as compared to LSB bits. The mechanism of LSB stenography is performed in this case however MSBs are used in place of LSBs.

\section{Cipher Bits}

This is another mechanism to ensure the safeguard of transmitted image over the career[10]. The image meant to be transmitted over the medium however before transmission image is encoded and cipher image is obtained. The key that can be public or private is also generated. This key is transmitted along with the image itself. At the other end decryption mechanism is implied to resolve the problem into desired image formats.

D. AES

Advanced encryption slandered can be used in order to provide encryption of images for security[11]. AES provide 128 bit encryption with 32 distinct segment formats. Keys are generated which are shared with sender and receiver. Keys are used to decode the image which is received at the destination end.

\section{IMAGE AUTHENTICATION}

This is the mechanism in which username and password is allocated to the image. In order to access the image username and password is required to be given[12]. The wrong password ensures de-allocation of resources. Image authentication is least secure since passwords can be easily guessed. In order to overcome this situation, image watermarking mechanism can be used. The proposed methodology is given in next section.

\section{PROPOSED METHODOLOGY}

Watermarking in existing literature is done by the use of discrete wavelet transformation[13], [14]. Advancement in terms of slant let transformation is giving better result in terms of parameters MSE and PSNR. MSE is obtained by the use of following equation

$$
M S E=\sum \frac{\left(X-X_{i}\right)^{2}}{n}
$$

Where $\mathrm{n} I$ the total number of pixels and $\mathrm{x}$ is the actual value of the result. $X_{i}$ is the result obtained after applying proposed mechanism.

Peak signal to noise ratio is obtained by the use of following equation

$$
\mathrm{PSNR}=10 * \log \left(\frac{\text { signal }}{\text { Noise }}\right)
$$

The flow of the proposed system is given in terms of the flowchart as

\section{PERFORMANCE ANALYSIS AND RESULTS}

Digital watermarking is a productive strategy to ensure copyright and responsibility for data. Digital watermarking is the strategy for inserting digital data in any type of sight and sound information, for example, picture, sound, video, and so forth. It is a technique for concealing one mystery 
message in another message. In prior days watermarks were utilized as trademark or logo for showing the responsibility for particular product. But in conventional techniques for digital picture watermarking, the surface of unique picture gets mutilated pretty much.

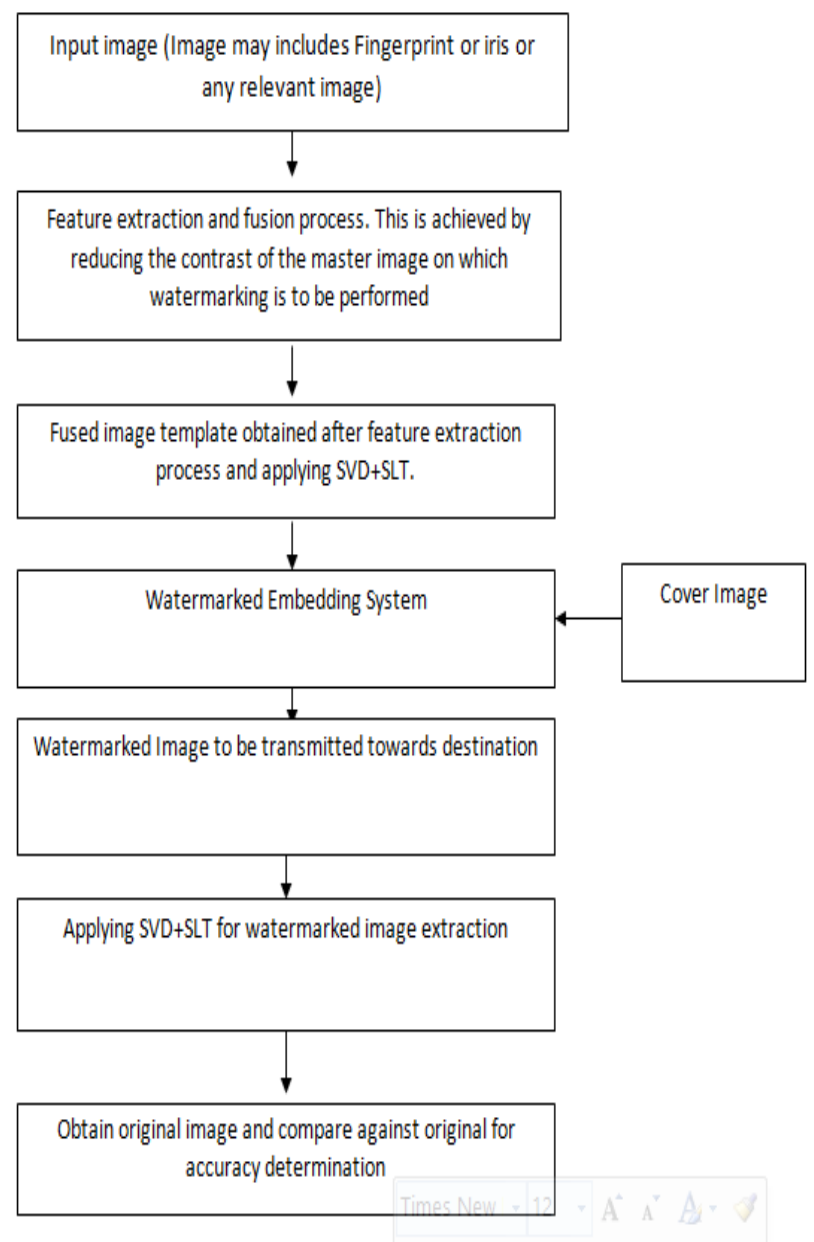

Fig. 1 Flowchart of Proposed Methodology

TABLE I COMPARISON OF MEAN SQUARE ERROR

\begin{tabular}{|l|c|c|}
\hline Image set & MSE Existing & MSE Proposed \\
\hline Image1 & 14.0869 & 7.04345 \\
\hline Image 2 & 15.7442 & 7.87209 \\
\hline Image3 & 132.03 & 66.015 \\
\hline Image4 & 14.0869 & 7.04345 \\
\hline Image5 & 31.7646 & 15.8823 \\
\hline
\end{tabular}

In proposed system noise is handled by component capable of introducing clarity within the image though filtering. In the wake of getting the clearness watermarking is forced. The picture information introduced to the reproduction is of .jpg and .png type. Results as far as MSE and PSNR is acquired the coveted reproduction.

Plots of result from the comparison table is as under

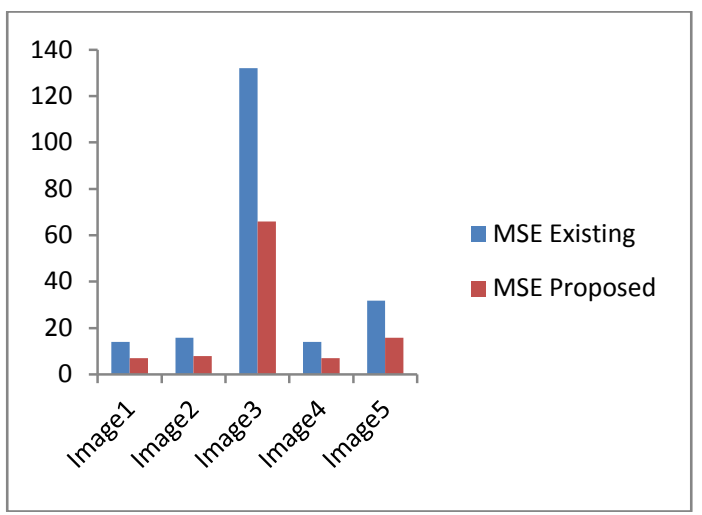

Fig. 2 Plots of MSE

TABLE II COMPARISON IN TERMS OF PEAK SIGNAL TO NOISE RATIO

\begin{tabular}{|l|c|c|}
\hline Image set & PSNR Existing & PSNR Proposed \\
\hline Image1 & 18.3383 & 39.6869 \\
\hline Image 2 & 18.0968 & 39.2039 \\
\hline Image3 & 13.479 & 29.9684 \\
\hline Image4 & 18.3383 & 39.6869 \\
\hline Image5 & 16.5727 & 36.1557 \\
\hline
\end{tabular}

Plots of PSNR with existing and proposed mechanism are given as under

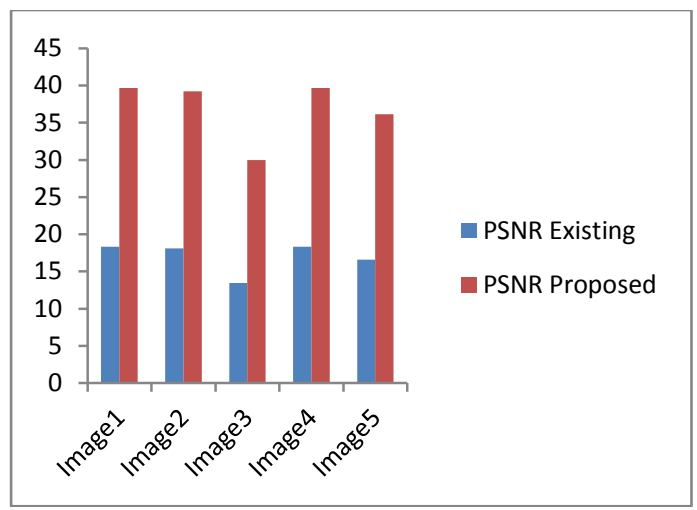

Fig. 3 Plot of PSNR

Result in terms of PSNR and MSE of proposed system is better proving the worth of the study.

\section{CONCLUSION}

Data Transmission through digital media is regular now days. As transmission through digital media is expanding, so does the assaults. At the season of transmission this data may get influenced by clamor, or some outsider tries to get that data and alter it. This can be forestalled utilizing digital watermarks. The sender who needs to send mystery or private picture to some other individual will install the mystery picture in another picture with the assistance of a key and send it through the Internet. The beneficiary will get that picture and concentrates the concealed watermark from that picture with the assistance of the mutual key. 
Security of data and picture will be of prime concern. Improving security is proficient by the utilization of number of systems for this reason encryption and unscrambling instruments are fundamental. Encryption is usually performed on content data the scrambled content is normally known as figure content. The programmers may assault the encoded data since encryption systems are usually utilized. So as to upgrade the security watermark appears. The proposed approach improving the security by presenting lucidity of picture encryption and decoding through inclination let changes. The SLT decrease the span of the picture by disintegrating it. By doing as such LSB and MSB bits of the picture can undoubtedly be obliged. The outcomes acquired through the proposed approach are superior to the current one.

\section{REFERENCES}

[1] S. Sahar Afshan Indrabi and Sheenam, "Watermarking Digital Images: A Hybrid Approach", International Journal of Advanced Research in Computer Science and Software Engineering, Vol. 5, No. 5, pp. 1778-1785, 2015.

[2] P. Parmar and N. Jindal, "Image Security with Integrated Watermarking and Encryption 11 2", Vol. 9, No. 3, pp. 24-29, 2014.

[3] T. Bathinda, "Invisible Video Multiple Watermarking Using Optimized Techniques", 2016.
[4] R. T. Mohammed and B. E. Khoo, "Image Watermarking Using Slantlet Transform", ISIEA 2012 - 2012 IEEE Symp. Ind. Electron. Appl., pp. 281-286, 2012.

[5] R. K. Sheth and V. V. Nath, "Secured Digital Image Watermarking with Discrete Cosine Transform and Discrete Wavelet Transform Method”, Int. Conf. Adv. Comput. Commun. Autom., pp. 1-5, 2016.

[6] R. V Mahule, "Analysis of Image Security Techniques using Digital Image Watermarking in Spatial Domain", No. Nckite, pp. 19-26, 2015.

[7] Z. J. Xu, Z. Z. Wang and Q. Lu, "Research on Image Watermarking Algorithm based on DCT", Vol. 10, pp. 1129-1135, 2011.

[8] U. Islam, F. Khalid, M. Shah, Z. Khan, T. Mahmood, A. Khan, U. Ali and M. Naeem, "An Improved Image Steganography Technique Based on MSB Using Bit Differencing", 6th Int. Conf. Innov. Comput. Technol. INTECH 2016, pp. 265-269, 2017.

[9] V. Saravanan and A. Neeraja, "Security Issues in Computer Networks and Stegnography", 7th Int. Conf. Intell. Syst. Control. ISCO 2013, pp. 363-366, 2013.

[10] P. Singhai and A. Shrivastava, "An Efficient Image Security Mechanism Based on Advanced Encryption Standard", No. 13, 2015.

[11] S. S. Gonge, "An Integration of SVD Digital Image Watermarking with AES Technique for Copyright Protection and Security of Bank Cheque Image", pp. 769-778, 2016.

[12] Q. Chen, H. Hu and J. Xu, "Authenticated Online Data Integration Services", pp. 167-181.

[13] J. Singh and A. K. Patel, "An Effective Telemedicine Security Using Wavelet Based Watermarking”, pp. 2-7, 2016.

[14] M. Rizal, M. Isa and S. Aljareh, "A Watermarking Technique to Improve the Security Level in Face Recognition Systems", Multimed. Tools Appl., 2016. 